\title{
A quantitative analysis of lymphatic vessels in human breast cancer, based on LYVE-I immunoreactivity
}

\author{
T Kato*, I,2 , R Prevo ${ }^{3}$, G Steers ${ }^{2}$, H Roberts ${ }^{2}$, RD Leek², T Kimura', S Kameoka', T Nishikawa ${ }^{4}$, M Kobayashi ${ }^{5}$, \\ DG Jackson ${ }^{3}$, AL Harris ${ }^{6}, K^{\prime}$ Gatter ${ }^{2}$ and F Pezzella ${ }^{2}$ \\ 'Department of Surgery II, School of Medicine, Tokyo Women's Medical University, 8-I Kawadacho, Shinjuku-ku, Tokyo I 62-8666, Japan; ${ }^{2}$ Cancer \\ Research UK Tumor Pathology Group, Nuffield Department of Clinical Laboratory Sciences, University of Oxford, John Radcliffe Hospital, Headington, \\ Oxford OX3 9DU, UK; ${ }^{3}$ MRC Human Immunology Unit, Weatherall Institute of Molecular Medicine, John Radcliffe Hospital, Headington, Oxford OX3 \\ 9DS, UK; ${ }^{4}$ Department of Surgical Pathology, School of Medicine, Tokyo Women's Medical University, 8-I Kawadacho, Shinjuku-ku, Tokyo I 62-8666, \\ Japan; ${ }^{5}$ Department of Pathology, School of Medicine, Tokyo Women's Medical University, 8-I Kawadacho, Shinjuku-ku, Tokyo I 62-8666, Japan; ${ }^{6}$ Cancer \\ Research UK Molecular Oncology Laboratory, Institute of Molecular Medicine, John Raddliffe Hospital, Headington, Oxford OX3 9DU, UK
}

This study was undertaken to determine the highly sensitive method for detecting tumour lymphatic vessels in all the fields of each slide (LV), lymphatic microvessel density (LMVD) and lymphatic vessel invasion (LVI) and to compare them with other prognostic parameters using immunohistochemical staining with polyclonal (PCAB) and monoclonal antibodies (MCAB) to the lymphatic vessel endothelial hyaluronan receptor-I (LYVE-I), and the pan-endothelial marker factorVIII in a series of 67 human breast cancers. In all LYVE-I-stained sections, LV (some of which contained red blood cells) were frequently found localised in extralobular stroma, dermis, connective tissue stroma and adjacent to artery and vein, but were rare within the intralobular stroma or the tumour body (3/67 cases) or areas of widespread invasion. In contrast small blood vessels were observed in intra- and extralobular stroma in the factor VIII-stained sections. Quantitation of vessel numbers revealed that LYVE-I/PCAB detected a significantly larger number of LV than either H\&E or LYVE-I/MCAB $(P<0.000 I)$. LYVE-I/PCAB detected LVI in 25/67 cases (37.3\%) and their presence was significantly associated with both lymph node metastasis $\left(\chi^{2}=4.698, P=0.0248\right)$ and unfavourable overall survival $(O S)(P=0.0453)$, while not relapse- free survival (RFS) $(P=0.2948)$. LMVD had no influence for RFS and OS $(P=0.4879, P=0.1463$, respectively). Our study demonstrates that immunohistochemistry with LYVE-I/PCAB is a highly sensitive method for detecting tumour LV/LVI in breast cancer and LVI is a useful prognostic indicator for lymphatic tumour dissemination.

British Journal of Cancer (2005) 93, | |68-| |74. doi:I0.1038/sj.bjc.6602844 www.bjcancer.com

Published online 25 October 2005

(c) 2005 Cancer Research UK

Keywords: breast cancer; lymphangiogenesis; lymphatic microvessel density; lymphatic vessel invasion; lymphatic tumour dissemination; LYVE-I

The common pathways of cancer cell dissemination are via the lymphatics and the bloodstream. It has been well established that angiogenesis is necessary for tumour growth and haematogenous metastasis (Weidner et al, 1991; Horak et al, 1992; Fox et al, 1995; Kato et al, 2001). However, the role of the lymphatic system is less understood. Some investigators have concluded that it is impractical to distinguish between the blood and lymphatic vessel systems as independent routes of tumour dissemination because they are so interrelated (Fisher and Fisher, 1966). Our previous studies challenge this by demonstrating that differences between blood vessel invasion (BVI) and lymphatic vessel invasion (LVI) in breast cancer can be distinguished by using antibodies against factor VIII related antigen (Kato et al, 2002, 2003). However, the distinction between lymphatic vessels and blood vessels was sometimes difficult to determine and hence arbitrary. As yet there

*Correspondence: Dr T Kato, Onuma Hospital, 2- I0-2, Higashimizumoto, Katsushika-ku, Tokyo I25-0033, Japan; E-mail: t-kato@bd5.so-net.ne.jp Received 20 July 2005; revised 8 September 2005; accepted 28 September 2005; published online 25 October 2005 have been no specific immunohistochemical markers available that allow the identification of lymphatic vessels. Recent studies have proposed a number of potential lymphatic-specific markers, including the lymphatic vessel endothelial hyaluronan receptor-1 (LYVE-1) (Banerji et al, 1999; Jackson, 2004), Prox-1 (Wigle and Oliver, 1999), podoplanin (Breiteneder-Geleff et al, 1997), and vascular endothelial growth factor receptor-3 (VEGFR-3) (Kukk et al, 1996; Lymboussaki et al, 1999; Jackson, 2001) and D2-40 (Kahn et al, 2002).

There has been debate in the literature as to whether lymphatic vessels exist within tumours (Leu et al, 2000; Nathanson et al, 2000; Padera et al, 2000; Birner et al, 2001; Mandriota et al, 2001; Schoppmann et al, 2001; Skobe et al, 2001; Stacker et al, 2001; Beasley et al, 2002; Dadras et al, 2003; Hall et al, 2003; Maula et al, 2003; Straume et al, 2003; Williams et al, 2003); whether tumours induce lymphangiogenesis (Leu et al, 2000; Nathanson et al, 2000; Sleeman, 2000; Mandriota et al, 2001; Schoppmann et al, 2001; Skobe et al, 2001; Stacker et al, 2001; Beasley et al, 2002; Williams et al, 2003); whether lymphangiogenesis or dilated pre-existing lymphatic vessels increase the probability of lymphatic tumour dissemination (Leu et al, 2000; Padera et al, 2000; Sleeman, 2000; 
Dadras et al, 2003; Straume et al, 2003; Bono et al, 2004); and whether cancer cells require active intravasation (Hartveit, 1990; Gunningham et al, 2000; Kinoshita et al, 2001; Schoppmann et al, 2001).

In this study, we used both polyclonal and monoclonal antibodies to the lymphatic-specific marker LYVE-1, a homologue of the CD44 hyaluronan receptor (Banerji et al, 1999; Jackson, 2003; Jackson, 2004) to identify lymphatic vessels in breast tumours. In parallel, we also looked at haematoxylin and eosin (H\&E) and factor VIII-related antigen staining (FVIII). We determined the presence and distribution of lymphatic vessels and examined the relationships of LVI and lymph-node metastases in a retrospective series of 67 human breast carcinomas.

\section{PATIENTS AND METHODS}

\section{Tumour collection}

In all, 67 consecutive unselected patients who underwent breast cancer surgery at the Tokyo Women's Medical University Hospital between January 1991 and December 1991 were included in the present study. To be included, the patients (all female) had to have primary, unilateral, breast cancer and no other malignancy. Clinical and pathological data are listed in Table 1. Estrogen and progesterone receptor (ER and $\mathrm{PR}$ ) content were determined biochemically using the dextran-coated charcoal (DCC) method. Tumours were classified as estrogen receptor or progesterone receptor-positive if the content exceeded $5 \mathrm{fmol} \mu \mathrm{g}^{-1}$.

Table I Clinicopathologic characteristics of 67 patients

\begin{tabular}{lc}
\hline Characteristics & No. of patients (\%) \\
\hline Patients enrolled & 67 \\
Age (years) & 49 \\
Median & $30-86$ \\
Range & \\
Menopausal status & \\
Pre & $36(53.7)$ \\
Post & $31(46.3)$ \\
Clinical tumor size, T & \\
TI & \\
T2 & $26(38.8)$ \\
T3 & $34(50.7)$ \\
& $7(10.5)$ \\
Lymph-node status & \\
Negative & \\
Positive & $43(64.2)$ \\
Unknown & $20(29.9)$ \\
ER & $4(5.9)$ \\
Negative & \\
Positive & $29(43.3)$ \\
Unknown & $35(52.2)$ \\
PR & $3(4.5)$ \\
Negative & \\
Positive & $38(56.7)$ \\
Unknown & $26(38.8)$ \\
Histological classification & $3(4.5)$ \\
Noninvasive ductal carcinoma & \\
Infiltrating ductal carcinoma & \\
Infiltrating lobular carcinoma & $5(7.5)$ \\
Others & $1(85.0)$ \\
\hline ER estron recept & $4(6.0)$ \\
\hline
\end{tabular}

$\mathrm{ER}=$ estrogen receptor; $\mathrm{PR}=$ progesteron receptor.

\section{Immunocytochemical techniques}

Serial sections were prepared from representative formalin-fixed and paraffin-embedded tissue blocks from this series of breast cancer. Tissue samples of $5 \mu \mathrm{m}$ thick sections stained with haematoxylin and eosin ( $\mathrm{H} \& \mathrm{E})$ were assessed histopathologically and were used to select the maximal area of all the cut surfaces of the tumour that included the invasive components. Immunostains for FVIII-related antigen were performed on paraffin sections using the streptavidin-biotin-immunoperoxidase method as previously described (Kato et al, 2002, 2003). Briefly, formalin-fixed, paraffin-embedded sections were dewaxed in $100 \%$ Citroclear, rehydrated through graded $100 \%$ industrial methylated spirit (IMS) series, and immunostaining was performed using a polyclonal antibody (von Willebrand factor, Dako, Copenhagen, Denmark) applied at $1: 200$ for $1 \mathrm{~h}$ at room temperature. Technical details of the polyclonal and monoclonal LYVE-1 staining are outlined in Tables 2 and 3. A normal human tonsil served as a

Table 2 The method for polyclonal antibody against LYVE antigen

\begin{tabular}{|c|c|}
\hline । & Deparaffinise sections in $100 \%$ citroclear for $10 \mathrm{~min}$ \\
\hline 2 & $\begin{array}{l}\text { Rehydrate through graded } 100 \% \text { industrial methlated spirits (IMS) } \\
\text { series for } 5 \mathrm{~min}\end{array}$ \\
\hline 3 & Predigest with $0.1 \%$ Protease for $5 \mathrm{~min}$ \\
\hline 4 & $\begin{array}{l}\text { Abolish endogenous peroxidase activity with } 3 \% \text { hydrogen } \\
\text { peroxide for } 20 \mathrm{~min}\end{array}$ \\
\hline 5 & Leave slides to tap water for 5 min \\
\hline 6 & Wash in phosphorate-buffered saline (PBS, pH 7.0) \\
\hline 7 & $\begin{array}{l}\text { Suppress nonspecific background staining with } 5 \% \text { normal human } \\
\text { serum for } 15 \mathrm{~min}\end{array}$ \\
\hline 8 & $\begin{array}{l}\text { Apply primary antibody }(1: 600 \text { diluted LYVE-I polyclonal antibody } \\
\text { in PBS) for I h at room temperature }\end{array}$ \\
\hline 9 & Wash in PBS for $5 \mathrm{~min}$ \\
\hline 10 & $\begin{array}{l}\text { Apply secondary antibody (DAKO anti-rabbit envision HRP } \\
\text { polymer) for } 30 \mathrm{~min} \text { at room temperature }\end{array}$ \\
\hline | | & Wash in PBS for $5 \mathrm{~min}$ \\
\hline 12 & $\begin{array}{l}\text { Apply } 0.05 \% 3,3^{\prime} \text {-diaminobenzidine tetrahydrochloride (DAB) } \\
\text { substrate provided in envision kit for } 4 \mathrm{~min}\end{array}$ \\
\hline 13 & Wash in distilled water \\
\hline 14 & Counter-staining by hematoxylin \\
\hline 15 & Aquamount \\
\hline
\end{tabular}

LYVE: lymphatic vessel endothelial hyaluronan receptor-1.

Table 3 The method for monoclonal antibody against LYVE antigen

\begin{tabular}{|c|c|}
\hline I & Deparaffinise sections in $100 \%$ citroclear for 10 min \\
\hline 2 & $\begin{array}{l}\text { Rehydrate through graded } 100 \% \text { industrial methlated spirits (IMS) series } \\
\text { for } 5 \mathrm{~min}\end{array}$ \\
\hline 3 & $\begin{array}{l}\text { Antigen retrieve; microwave in Dako target antigen retrieval buffer } \\
\text { diluted } 1: 10 \text { at } 95-100^{\circ} \mathrm{C} \text { for } 40 \mathrm{~min}\end{array}$ \\
\hline 4 & $\begin{array}{l}\text { Wash in water, rinse distilled water, then transfer tris-buffered saline } \\
\text { (TBS) }\end{array}$ \\
\hline 5 & $\begin{array}{l}\text { Abolish endogenous peroxidase activity with peroxidase block from } \\
\text { Dako envision kit for } 5 \mathrm{~min}\end{array}$ \\
\hline 6 & Wash in TBS for $5 \mathrm{~min}$ \\
\hline 7 & $\begin{array}{l}\text { Apply primary antibody (LYVE-I monoclonal antibody diluted I : } 2 \text { with } \\
0.1 \% \text { bovine serum albumin in TBS) at } 4^{\circ} \mathrm{C} \text { over night }\end{array}$ \\
\hline 8 & Wash in TBS for $5 \mathrm{~min}$ \\
\hline 9 & $\begin{array}{l}\text { Apply secondary antibody (DAKO anti-mouse envision HRP polymer) } \\
\text { for } 30 \mathrm{~min} \text { at room temperature }\end{array}$ \\
\hline 10 & Wash in TBS for $5 \mathrm{~min}$ \\
\hline 11 & $\begin{array}{l}\text { Apply } 0.05 \% 3,3^{\prime} \text {-diaminobenzidine tetrahydrochloride (DAB) } \\
\text { substrate provided in envision kit for } 5 \mathrm{~min}\end{array}$ \\
\hline 12 & Wash in TBS, rinse distilled water \\
\hline 13 & Counter-staining by hematoxylin \\
\hline 14 & Aquamount \\
\hline
\end{tabular}

LYVE = lymphatic vessel endothelial hyaluronan receptor- I. 
positive control. Rabbit polyclonal antibody and mouse monoclonal antibody to LYVE-1 (LYVE-1/PCAB and LYVE-1/MCAB) were generated as described previously (Banerji et al, 1999; Cao et al, 2004).

\section{Assessment of lymphatic vessels}

HळE staining We defined as lymphatics those vessels lined by flattened endothelial cells, in the presence or absence of lymphocytes and absence of erythrocytes, in the stroma or adjacent to arteries and veins.

LYVE-1/PCAB. staining and $L Y V E-1 / M C A B$ staining Positive vessels were scored as lymphatic vessels. Staining intensity was assessed as follows; strong staining; moderate staining; weak staining (Figure 1A and B).

\section{Counting of lymphatic vessels and determination of lymphatic microvessel density (LMVD) and blood microvessel density (BMVD)}

Both the number and intensity of staining of the lymphatic vessels were evaluated. The intensity of staining and level of tissue damage were expressed as weak, moderate and strong. We defined as a lymphatic vessel the vessel, which have endothelium with immunopositivity and a vascular lumen. Mean lymphatic vessel count was determined by averaging the number of total lymphatic vessels in all the fields of each slide, including within the tumour or at the periphery of the tumour, at $\times 100$ or $\times 200$ magnification. Single brown-stained endothelial cells with a lumen were counted as individual lymphatic vessels, as shown in Figure 1C. The three most vascularised areas ('hot spots') were selected at low power magnification $(\times 40)$ and LMVD and BMVD were then determined by counting all LYVE-1/PCAB-immunostained or factor VIII related antigen stained vessels at $\times 200$ magnification. When the average number was higher than the median number of LYVE-1/PCAB or FVIII related antigen positive vessels, the cancer was considered to have a high LMVD or BMVD, otherwise a low LMVD or BMVD.

\section{Statistical analysis}

Statistical analysis of the data was performed with the Survival Tools for Statview-J 5.0. package (Abacus Concepts, Berkeley, CA, USA). For comparison of number of lymphatic vessel assessed by the three different staining methods, for association of LMVD and clinical or pathologic parameters and for the association of LVI and lymph-node status, Kruskal-Wallis test, Mann-Whitney $U$-test and $\chi^{2}$ test were used. The association of the numbers of lymphatic vessels in the LYVE-1/PCAB and those in LYVE-1/ MCAB stained sections was assessed by Pearson's correlation coefficient. We examined the univariate relationships between prognostic indicators and relapse-free survival (RFS) and overall survival (OS) by fitting Kaplan - Meier survival curves (Kaplan and Meier, 1958) to various levels of the prognostic indicators.

\section{RESULTS}

Both the polyclonal and monoclonal anti LYVE-1 antibodies yielded specific and consistent staining of endothelial cells in the lymphatic vessels (Figure 1A and B). Many lymphatic vessels were frequently detected in dermis, connective tissue stroma (Figure 1A and $\mathrm{B}$ ), retro-mammary tissue, adjacent to artery and vein and extralobular stroma (Figure 1C). However, lymphatic vessels were rarely seen in intralobular stroma (Figure $1 \mathrm{C}$ ), intra-tumour tissue, areas of necrosis, adipose tissue (Figure $1 \mathrm{~A}$ and $\mathrm{B}$ ) and muscle. In contrast, in the FVIII-stained sections small blood vessels were observed in both intra- and extralobular stroma (Figure 1D). In addition to those findings many lymphatic vessels, which contained red blood cells were observed in H\&E, FVIII staining, LYVE-1/PCAB and LYVE-1/MCAB-stained sections. (Figure $1 \mathrm{E}-\mathrm{H})$. It was difficult to distinguish between lymphatic vessels and blood vessels by the finding of the presence or absence of erythrocytes in the lumen of vessels detected by $\mathrm{H} \& \mathrm{E}$ staining alone.

The mean and median (range) number of all lymphatic vessels is shown in Table 4. The total and the mean number of LYVE-1/ PCAB-immunostained lymphatic vessels were higher than that of the H\&E and LYVE-1/MCAB- stained lymphatic vessels. $(P<0.0001)$. Strong significant correlation was between the LYVE-1/PCAB-immunostained lymphatic vessels and LYVE-1/ MCAB-immunostained lymphatic vessels (Pearson's correlation coefficient $=0.815, P<0.0001)$. Median LMVD was 6.1 microvessels $\mathrm{mm}^{-2}$ (range $0-17.9$ vessels). A strong significant correlation was found between LMVD and LYVE-1/PCAB-immunostained lymphatic vessels (Pearson's correlation coefficient $=0.718$, $P<0.0001)$. There was no significant correlation between the LMVD and BMVD (Pearson's correlation coefficient $=0.021$, $P=0.8710)$. An inverse correlation was seen between histological grading and LMVD $(P=0.0434)$, while histological grading or menopausal status trended with the number of lymphatic vessels $(P=0.0712)$ or LMVD $(P=0.0944)$. There was no significant correlation between clinical tumour size, lymph-node status, LVI, or estrogen receptor and LMVD or the mean number of lymphatic vessels (Table 5). LVI was detected by H\&E, LYVE-1/PCAB and LYVE-1/MCAB staining in 23/67 cases (34.3\%), 25/67 cases $(37.3 \%)$ and $20 / 67$ cases (29.9\%), respectively. The lymph-node status or LVI detected by H\&E, LYVE-1/PCAB and LYVE-1/MCAB was not associated with the mean number of lymphatic vessels $(P=0.6413, \quad P=0.8339, \quad P=0.8884$ or $P=0.7412, \quad P=0.5759$; $P=0.8075$, respectively), but LVI detected by LYVE-1/PCAB was significantly associated with lymph-node status $\left(\chi^{2}=4.698\right.$, $P=0.0248$, Table 6). A significant difference in OS was found between patients with LVI or without LVI $(P=0.0453)$, while no significant difference in RFS $(P=0.2948)$. However, LMVD had no influence for OS and RFS ( $P=0.4879, P=0.1463$, respectively).

\section{DISCUSSION}

This manuscript has focused on the utility of different LYVE-1 antibodies as routine markers for detecting and quantitating lymphatic vessels in breast cancer. Our results confirm that both LYVE-1 polyclonal and monoclonal antibodies distinguish efficiently between lymphatic and blood vessels in pathological specimens. However, due to the greater dependence of LYVE-1 monoclonal antibodies on tissue fixation and antigen retrieval methods that result in partial antigen destruction, we have found that LYVE-1 polyclonal antibodies are suited to routine immunohistochemical staining applications. Furthermore, using immunohistochemical staining with LYVE-1 polyclonal antibodies we have shown that lymphatic invasion is positively associated with lymph node involvement and unfavorable OS.

Quantitation of tumour lymphatic vessels for the purpose of tumour staging has for decades been problematic. Although morphology can sometimes distinguish lymphatic vessels from blood vessels by the frequent absence of a basement membrane and lack of erythrocytes in the latter, neither is a reliable method for routine use. These considerations have hampered the reliable identification of tumour lymphatic vessels in routine histopathology. More recently, however, the development of specific markers such as the lymphatic hyaluronan receptor LYVE-1, the subject of this manuscript, has allowed many new experimental studies of tumour lymphatics to be initiated. To date, the majority of these studies have employed LYVE-1 polyclonal antibodies, requiring 

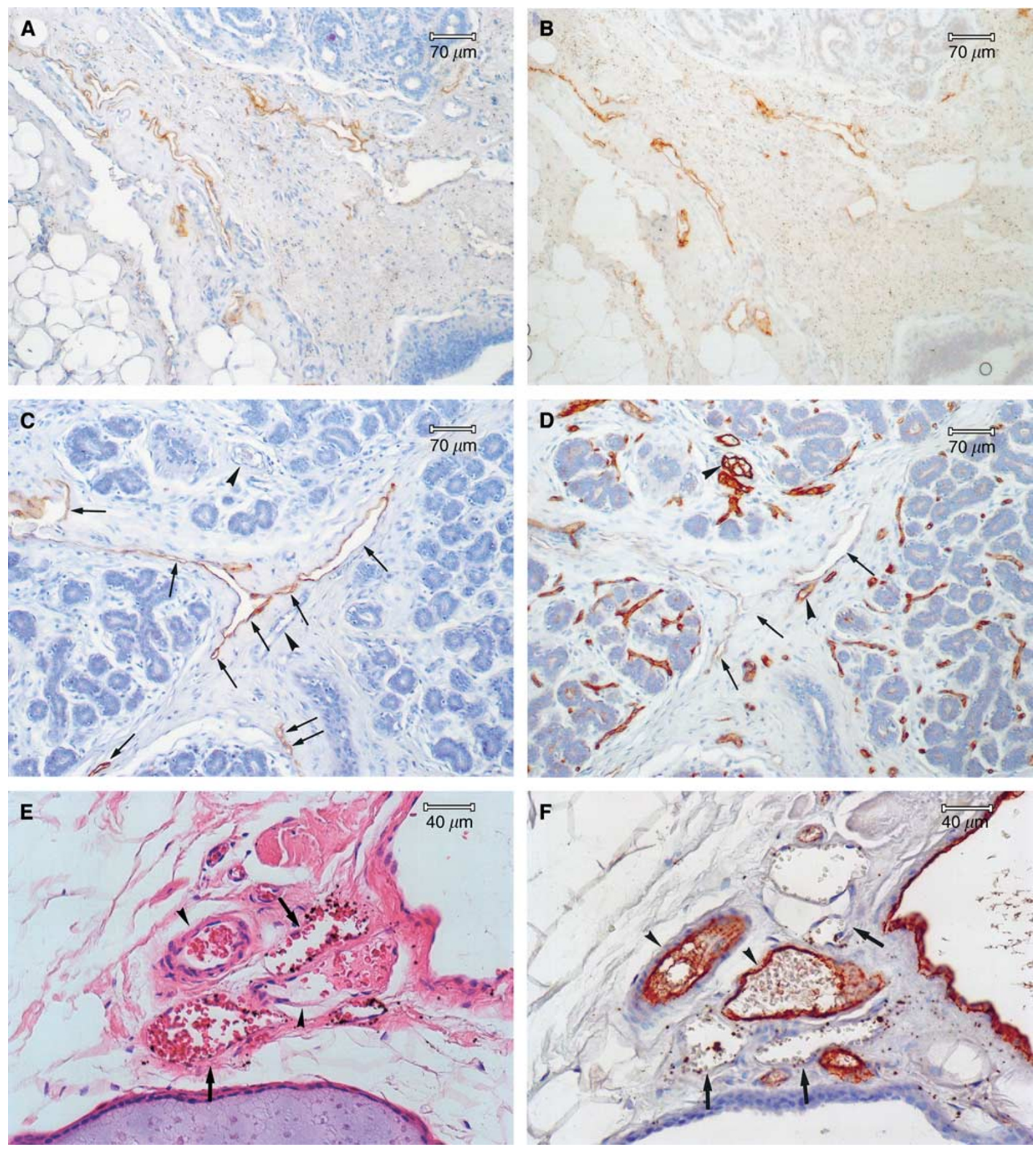

Figure I (A) Several LYVE-I/PCAB-positive lymphatic vessels are present in the connective tissue stroma (A: LYVE-I/PCAB staining, haematoxylin counter stain). (B) The monoclonal anti LYVE-I antibodies (LYVE-I/PCAB) yielded specific and consistent staining of endothelial cells in the lymphatic vessels (B: LYVE-I/MCAB staining, haematoxylin counter stain). (C) The arrows indicate lymphatic vessels, which are detected by LYVE-I/PCAB staining. We found nine lymphatic vessels in this field. Lymphatics are frequently found in extralobular stroma, but rarely seen in intralobular stroma. Blood vessels (arrowheads) are not stained by the staining (C: LYVE-I/PCAB staining, haematoxylin counter stain). (D) The arrowheads show blood vessels, which are stained by factor VIII related antigen staining. They are found in both intra- and extralobular stroma. The intensity of endothelial cells in the lymphatic vessels (arrows) in factor VIII related antigen-stained section is very faint, discontinuous and inconsistent (D: factor VIII-related antigen staining, haematoxylin counter stain). (E, F, G and $\mathbf{H}$ ) Serial sections were prepared for 4 kinds of staining. Some lymphatic vessels (arrows), which contained red blood cells, were observed in haematoxylin and eosin (H\&E), factor VIII related antigen, LYVE-I/PCAB and LYVE-I/MCAB-stained sections. Arrows indicate lymphatic vessels and arrowheads show blood vessels (E: H\&E staining; F: factor VIII related antigen staining; G: LYVE-I/PCAB staining; H: LYVE-I/MCAB staining, haematoxylin counter stain). (I) A lymphatic vessel with floating tumour cells (LVI) was found localised in the connective tissue stroma (I: LYVE-I/PCAB staining, haematoxylin counter stain). 

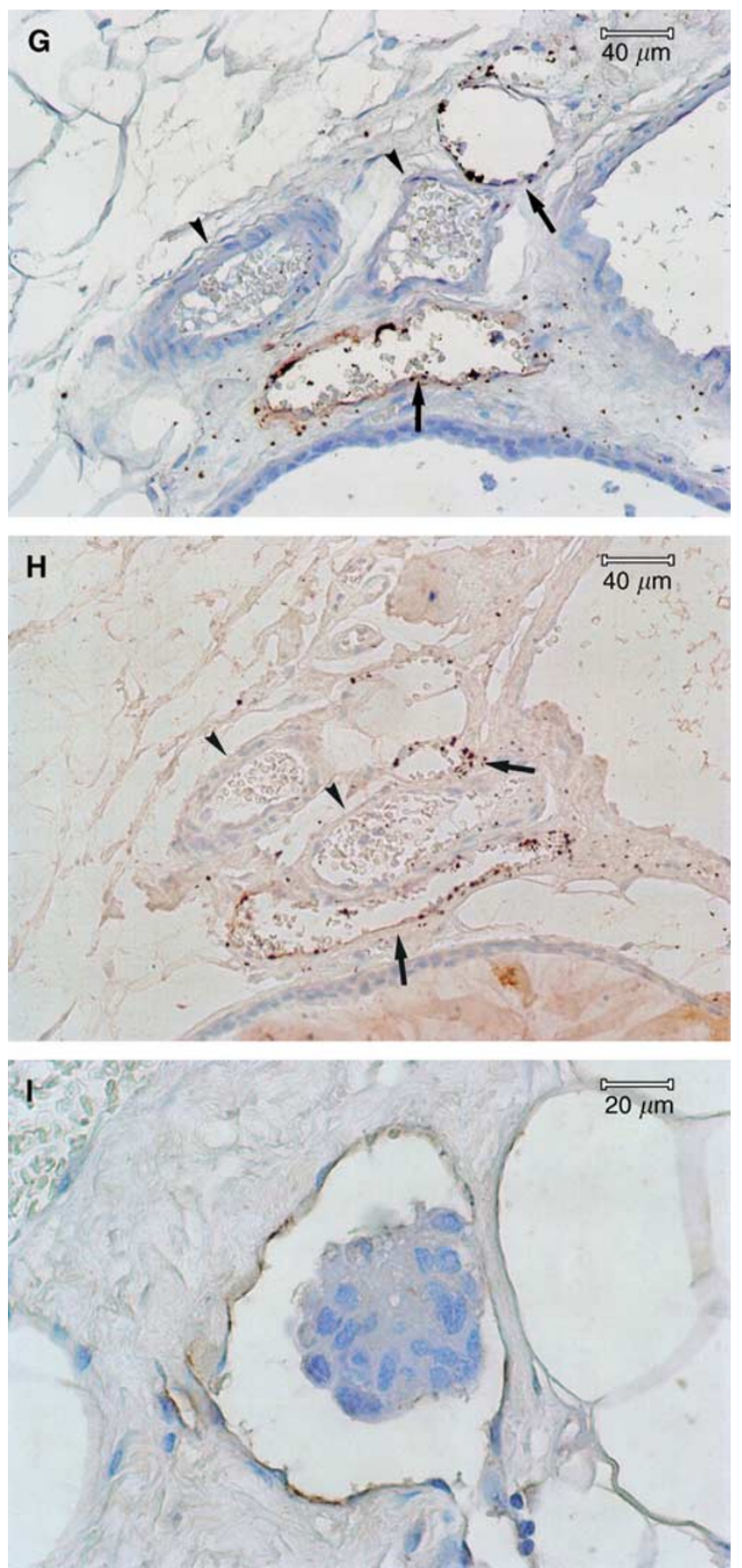

Figure I Continued.

microwave treatment or pressure cooking for antigen retrieval (Banerji et al, 1999; Beasley et al, 2002; Williams et al, 2003). In this present manuscript, we found that the intensity of many endothelial cells in the lymphatic vessels of either microwave or pressure cooking treatment stained with LYVE-1/PCAB was similar to that seen with protease retrieved tissue sections. However, some breast tissue sections in the former treatment were damaged, so it was difficult to observe the lymphatic vessels of all the fields in each slide. These results confirm that proteolytic enzyme treatment for LYVE-1/PCAB staining as well as for FVIII staining is more useful than either microwave or pressure cooking treatment in the human breast tissue. The LYVE-1/MCAB requires the use of microwave antigen retrieval to produce good staining
Table 4 Comparison of the three methods for detection of lymphatic vessels

\begin{tabular}{|c|c|c|c|c|}
\hline Methods of staining & H\&E & $\begin{array}{l}\text { LYVE-I/ } \\
\text { PCAB }\end{array}$ & $\begin{array}{l}\text { LYVE-II } \\
\text { MCAB }\end{array}$ & $P$-value \\
\hline \multicolumn{5}{|c|}{ Number of lymphatic vessels } \\
\hline Total number & 4274 & 17334 & 10919 & \\
\hline \multicolumn{5}{|l|}{ of 67 cases } \\
\hline Mean \pm s.d. & $63.8 \pm 64.4$ & $\begin{array}{c}258.7 \pm 219.1 \\
204\end{array}$ & $\begin{array}{c}163.0 \pm 155.7 \\
109\end{array}$ & $<0.0001$ \\
\hline Range & $4-372$ & $0-828$ & $2-575$ & \\
\hline \multicolumn{5}{|l|}{$\begin{array}{l}\text { Level of expression } \\
\text { (No. of patients) }\end{array}$} \\
\hline Weak & & $19(28.4 \%)$ & $25(37.3 \%)$ & \\
\hline Moderate & & 19 (28.4\%) & 25 (37.3\%) & \\
\hline Strong & & $29(43.2 \%)$ & $17(25.4 \%)$ & \\
\hline \multicolumn{5}{|l|}{$\begin{array}{l}\text { Level of tissue damage } \\
\text { (No. of patients) }\end{array}$} \\
\hline Weak & & $59(88.1 \%)$ & $5(7.5 \%)$ & \\
\hline Moderate & & $8(11.9 \%)$ & 48 (71.6\%) & \\
\hline Strong & & 0 & 14 (20.9\%) & \\
\hline
\end{tabular}

H\&E = hematoxylin and eosin; LYVE-I = lymphatic vessel endothelial hyaluronan receptor- $1 ; \mathrm{PCAB}=$ polyclonal antibody; $\mathrm{MCAB}=$ monoclonal antibody.

without background. The staining of lymphatic vessels in LYVE-1/ $\mathrm{MCAB}$-stained sections was occasionally more intense than that in LYVE-1/PCAB-stained sections (Figure $1 \mathrm{~A}$ and $\mathrm{B}$ ). However, because of the use of microwave antigen retrieval, section quality is often low so that the estimation of the total number of lymphatic vessels is less reliable than with LYVE-1/PCAB staining.

Previous studies have reported that all blood vessels that contain erythrocytes are negative for LYVE-1 supporting its specificity for lymphatics (Banerji et al, 1999; Williams et al, 2003). However, in this study, we observed some LYVE-1-positive vessels containing erythrocytes in both LYVE-1/PCAB and LYVE-1/MCAB-stained sections (Figure $1 \mathrm{E}-\mathrm{H}$ ). A prominent function of the lymphatic system is the provision of fluid drainage of lymphocytes, protein, colloid and foreign antigens from the tissues to the peripheral lymph nodes. Moreover lymphatic vessels act as a conduit for both migrating inflammatory cells and possibly erythrocytes from inflammatory tissue and parenchyma or stroma with haemorrhage. Hartveit has described tumour cells in the periductal lymphatic spaces being washed with the tide of interstitial fluid into the lymphatic network and into the lymphatic vessels (Hartveit, 1990). Therefore lymphatic vessels containing erythrocytes may well be observed from time to time especially in tumours. Based on similar findings in tumours by Padera the specificity of LYVE-1 for lymphatics has recently been questioned. This can be explained either by the presence of haemovascular-lymphovascular shunts (Clarijs et al, 2001; Abtahian et al, 2003), by leakiness of newly proliferating tumour blood capillaries that is high degree of fenestration, or rupture giving rise to $\mathrm{RBC}$ that enter the lymphatic vessels. It is possible that there are more of these in tumours further explaining our findings of erythrocytes in LYVE-1 positive vessels.

Several recent studies in animal models have reported that lymphatic vessels are frequently observed in the peripheral rim of the tumour, but not in the body of the tumour itself (Leu et al, 2000; Padera et al, 2000). However, others demonstrated the existence of intratumoural lymphatic vessels, in xenotransplanted breast tumours and fibrosarcomas in mice and in human head and neck cancers respectively (Skobe et al, 2001; Stacker et al, 2001; Beasley et al, 2002; Jackson, 2004). Most studies in human breast cancer described a similar peritumoural distribution (Nathanson et al, 2000; Schoppmann et al, 2001; Williams et al, 2003; Bono et al, 2004) which is largely supported by the current study which 
Table 5 Association of LMVD and other clinical and pathologic parameters

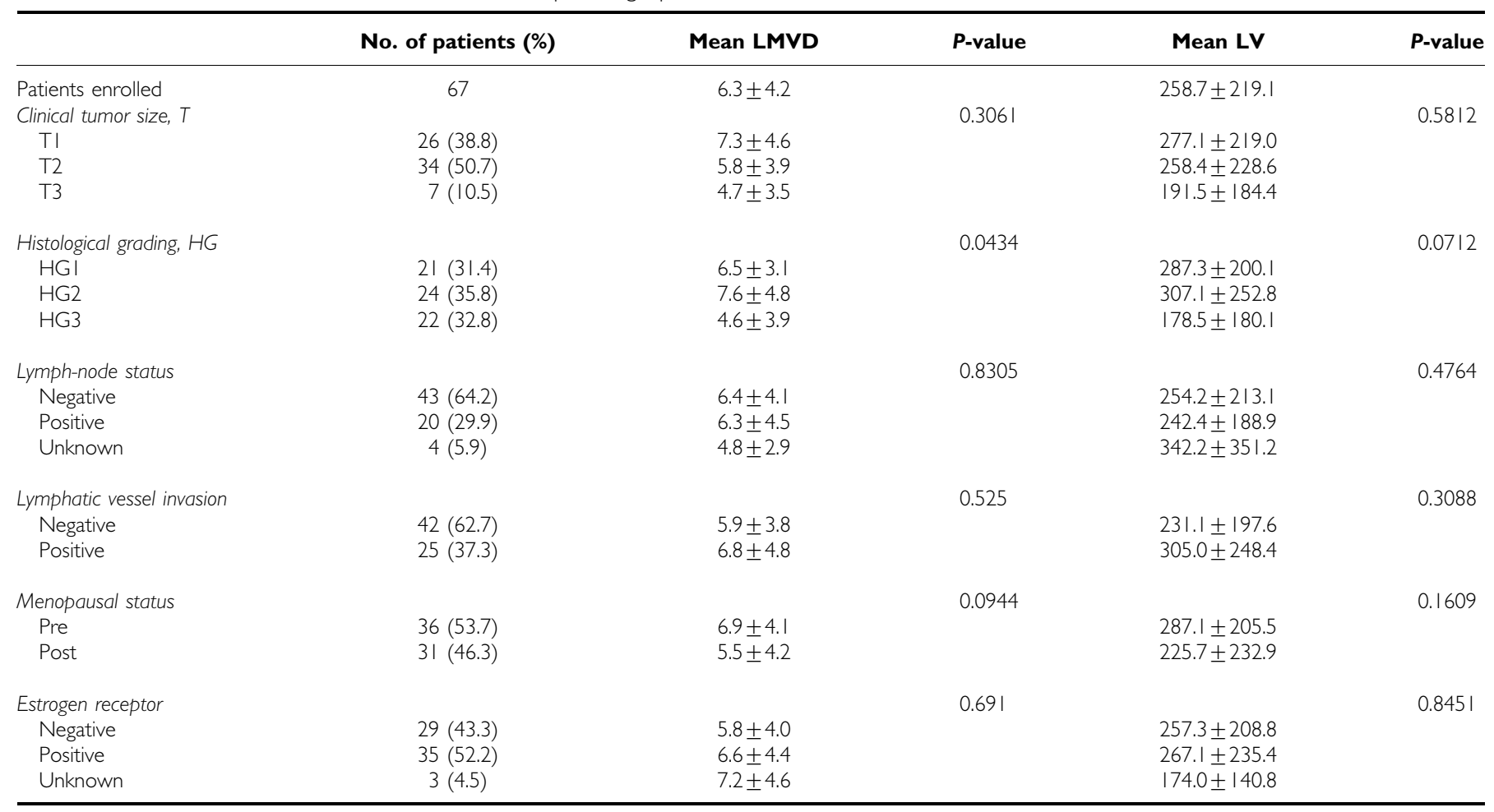

$L M V D=$ lympatic microvessel density; $L V=$ the number of total lymphatic vessels in all the fields of each slide.

Table 6 Association of LVI detected by three kinds of staining and lymph-node status

\begin{tabular}{|c|c|c|c|c|}
\hline \multirow[b]{2}{*}{$\begin{array}{l}\text { Methods of } \\
\text { staining }\end{array}$} & \multirow[b]{2}{*}{$\begin{array}{c}\text { No. of } \\
\text { patients }\end{array}$} & \multicolumn{2}{|c|}{ Lymph-node statusc } & \multirow[b]{2}{*}{$P$-value } \\
\hline & & $\begin{array}{c}\text { Negative } \\
\text { No. of } \\
\text { patients (\%) }\end{array}$ & $\begin{array}{c}\text { Positive } \\
\text { No. of } \\
\text { patients (\%) }\end{array}$ & \\
\hline LVI/H\&E & & & & 0.1293 \\
\hline Negative & 40 & $30(75.0)$ & $10(25.0)$ & \\
\hline Positive & 23 & $13(56.5)$ & $10(43.5)$ & \\
\hline LVI/LYVE-I/PCAB & & & & 0.0146 \\
\hline Negative & 39 & $31(79.5)$ & $8(20.5)$ & \\
\hline Positive & 24 & $12(50.0)$ & $12(50.0)$ & \\
\hline LVI/LYVE-I/MCAB & & & & 0.2457 \\
\hline Negative & 44 & $32(72.7)$ & $12(27.3)$ & \\
\hline Positive & 19 & II (57.9) & $8(42.1)$ & \\
\hline
\end{tabular}

$\mathrm{LVI}=$ lymphatic vessel invasion; $\mathrm{H} \& \mathrm{E}=$ hematoxylin and eosin; $\mathrm{LYVE}-\mathrm{I}=$ lymphatic vessel endothelial hyaluronan receptor-1; $P C A B=$ polyclonal antibody; $M C A B=$ monoclonal antibody. Four cases with unknown lymph-node status were excluded.

shows that lymphatic vessels are frequently found in the extralobular stroma and connective tissue stroma (Figure $1 \mathrm{~A}-\mathrm{C}$ ) but are rarely seen in the intralobular stroma and within the tumour itself. The reasons for this selective localisation of lymphatic vessels in tumours is unknown but one possibility is that they are collapsed in expanding tumours because of the high interstitial pressure. That would suggest that they are present, but difficult to detect because of their flattened morphology. In any case 'collapsed' is a subjective interpretation of appearance in histological sections. Even presumably fully functional lymphatic vessel in normal tissue can look 'collapsed'. The growth of tumour lymphatics is likely to be limited by availability of lymphangiogenic growth factors, related to infiltration by tumour associated macrophages (Williams et al, 2003), physical barriers to intratumoural lymphatic vessel growth (Mandriota et al, 2001) or inhibitory mechanisms as yet undefined, but perhaps similar to those that appear to prevent lymphatic vessel growth in the cornea (Cursiefen et al, 2002). Those lymphatic vessels which are present at the tumour periphery are considered to be pre-existing lymphatics rather than those induced by tumour lymphangiogenetic factors (Leu et al, 2000).

Recent studies have demonstrated that VEGFR-3 or D2-40 immunostained microvessels was associated with either node metastases (Nathanson et al, 2000) or BMVD (Choi et al, 2005) and a high peritumoural lymphatic vessel density is associated with a poor outcome in human breast cancer (Bono et al, 2004). On the other hand, other researchers reported that there was no significant correlation between LMVD and BMVD (Schoppmann et al, 2001; Bono et al, 2004), tumour size, histological grading or nodal status (Williams et al, 2003) and no significant difference between high lymphatic vessel density and low one for RFS and OS (Schoppmann et al, 2004). The results of our study suggest that an inverse correlation was seen between LMVD and histological grading, while there was no significant correlation between LMVD and lymph-node status or LVI and LMVD was not associated with a poor outcome. As there was no significant correlation between LMVD and BMVD, the genesis of lymphatic vessels might be different from that of blood vessels (Schoppmann et al, 2001). It is tempting to speculate that as lymphatics in tumour with increased aggressiveness could be excluded and destroyed by cancer (Williams et al, 2003), LMVD has no influence for lymphatic tumour dissemination.

The current study has used LYVE-1 staining to increase the accuracy and rate of detection of LVI since using this method makes it easy to distinguish from BVI (Figure 1I). In recent human studies the rate of LVI fell to within the range of 13.3 and $53.3 \%$ (Schoppmann et al, 2001; Williams et al, 2003), while the rate of 
LVI detected by LYVE-I/PCAB staining was seen in 25/67 cases $(37.3 \%)$ in this study, and the potential importance of measuring the LVI is that it has been strongly associated with the presence of

\section{REFERENCES}

Abtahian F, Guerriero A, Lu M-M, Zhou R, Mocsai A, Myers EE, Huang B, Jackson DG, Ferrari VA, Tybulewicz V, Lowell CA, Lepore JJ, Koretzky GA, Kahn ML (2003) Separation of blood and lymphatic vascular networks is regulated by the haematopoietic signalling proteins SLP-76 and SYK. Science 299: 247-251

Banerji S, Ni J, Wang SX, Clasper S, Su J, Tammi R, Jones M, Jackson DG (1999) LIVE-1, a new homologue of the CD44 glycoprotein, is a lymphspecific receptor for hyaluronan. J Cell Biol 144: 789-801

Beasley NJP, Prevo R, Banerji S, Leek R, Moore J, von Trappen P, Cox G, Harris AL, Jackson DG (2002) Lymphangiogenesis is linked to tumour metastasis in head and neck cancer. Cancer Res 62: 1315-1320

Birner P, Schindl M, Obermair A, Breitenecker G, Kowalski H, Oberhuber G (2001) Lymphatic microvessel density as a novel prognostic factor in early-stage invasive cervical cancer. Int J Cancer 95: 29-33

Bono P, Wasenius V-M, Lundin J, Jackson DG, Joensuu H (2004) High LYVE-1 positive lymphatic vessel numbers are associated with poor outcome in breast cancer. Clin Cancer Res 10: 7144-7149

Breiteneder - Geleff S, Matsuki K, Soleiman A, Meraner P, Poczewski H, Kalt R, Schaffner G, Kerjaschki D (1997) Podoplanin novel 43-kd membrane protein of glomerular epithelial cells, is down-regulated in puromycin nephrosis. Am J Pathol 151: 1141 -1152

Cao R, Bjorndahl M, Religa P, Garvin S, Galter D, Clasper S, Meister B, Ikomi F, Hansen AJ, Dissing S, Ohhashi T, Jackson DG, Cao Y (2004) PDGF-BB induces intratumoral lymphangiogenesis and promotes lymphatic metastasis. Cancer Cell 6: 205-226

Choi WWL, Lewis MM, Lawson D, Yin-Goen Q, Birdsong GG, Cotsonis GA, Cohen C, Young AN (2005) Angiogenic and lymphangiogenic microvessel density in breast carcinoma: correlation with clinicopathologic parameters and VEGF-family gene expression. Modern Pathol 18: $143-152$

Clarijs R, Ruiter DJ, de Waal RM (2001) Lymphangiogenesis in malignant tumours: does it occur? J Pathol 193: $143-146$

Cursiefen C, Schlotzer-Schrehardt U, Kuchle M, Sorokin L, BreitenederGeleff S, Alitalo K, Jackson D (2002) Lymphatic vessels in vascularized human corneas: immunohistochemical investigation using LYVE-1 and podoplanin. Invest Ophthalmol Vis Sci 43: 2127-2135

Dadras S, Paul T, Bertoncini J, Brown LF, Muzikansky A, Jackson DG, Ellwanger U, Garbe C, Mihm MC, Detmar M (2003) Tumor lymphangiogenesis: A novel prognostic indicator for cutaneous melanoma metastasis and survival. Am J Pathol 162: 1951 - 1960

Fisher B, Fisher ER (1966) The inter-relationship of hematogenous and lymphatic tumour cell dissemination. Surg Gynecol Obstet 122: 791-798

Fox SB, Leek RD, Weekes MP, Whitehouse RM, Gatter KG, Harris A (1995) Quantitation and prognostic value of breast cancer angiogenesis: Comparison of microvessel density, Chalkley count, and computer image analysis. J Pathol 177: 275-283

Gunningham S, Currie J, Han C, Robinson BA, Scott PAE, Harris AL, Fox SB (2000) The short form of the alternatively spliced flt-4 but not its ligand vascular endothelial growth factor $\mathrm{C}$ is related to lymph node metastasis in human breast cancer. Clin Cancer Res 6: 4278-4286

Hall FT, Freeman JL, Asa SL, Jackson DG, Beasley NJ (2003) Intratumoral lymphatics and lymph node metastases in papillary thyroid carcinoma. Arch Otolaryngol Head Neck Surg 129: 716-719

Hartveit F (1990) Attenuated cells in breast stroma: the missing lymphatic system of the breast. Histopathology 16: $533-543$

Horak ER, Leek R, Klenk N, Lejeune S, Smith K, Stuart N, Greenall M, Stepniewska K, Harris A (1992) Angiogenesis, assessed by platelet/ endothelial cell adhesion molecule antibodies, as indicator of node metastases and survival in breast cancer. Lancet 340: 1120-1124

Jackson DG (2001) New Markers for the study of Lymphangiogenesis. Anticancer Res 21: $4279-4283$

Jackson DG (2003) The Lymphatics revisited: New perspectives from the hyaluronan receptor LYVE-1. Trends Cardiovasc Med 13: 1-7

Jackson DG (2004) Biology of the lymphatic marker LYVE-1 and applications in research into lymphatic trafficking and lymphangiogenesis. APMIS 112: 526-538

Kahn HJ, Bailey D, Marks A (2002) Monoclonal antibody D2-40, a new marker of lymphatic endothelium, reacts with Kaposi's sarcoma and a subset of angiosarcomas. Mod Pathol 15: 434-440 lymph-node metastases and unfavorable OS in human breast cancer (Schoppmann et al, 2004). The results in this present study support that suggestion.
Kaplan EL, Meier P (1958) Non-parametric estimation from incomplete observations. J AmStat Assoc 53: $457-481$

Kato T, Kameoka S, Kimura T, Nishikawa T, Kobayashi M (2002) Blood vessel invasion as a predictor of long-term survival for Japanese patients with breast cancer. Breast Cancer Res Treat 73: 1-12

Kato T, Kameoka S, Kimura T, Nishikawa T, Kobayashi M (2003) The combination of angiogenesis and blood vessel invasion as a prognostic indicator in primary breast cancer. Br J Cancer 88: $1900-1908$

Kato T, Kameoka S, Kimura T, Soga N, Abe Y, Nishikawa T, Kobayashi M (2001) Angiogenesis as a predictor of long-term survival for 377 Japanese patients with breast cancer. Breast Cancer Res Treat 70: 65-74

Kinoshita J, Kitamura K, Kabashima A, Saeki H, Tanaka S, Sugimachi K (2001) Clinical significance of vascular endothelial growth factor-C (VEGF-C) in breast cancer. Breast Cancer Res Treat 66: 159-164

Kukk E, Lymboussaki A, Taira S, Kaipainen A, Jeltsch M, Joukov V, Alitalo K (1996) VEGF-C receptor binding and pattern of expression with VEGFR-3 suggests a role in lymphatic vascular development. Development 122: $3829-3837$

Leu AJ, Berk DA, Lymboussaki A, Alitalo K, Jain RK (2000) Absence of functional lymphatics within a murine sarcoma: a molecular and functional evaluation. Cancer Res 60: 4324-4327

Lymboussaki A, Olofsson B, Eriksson U, Alitalo K (1999) Vascular endothelial growth factor (VEGF) and VEGF-C show overlapping binding sites in embryonic endothelia and distinct sites in differentiated adult endothelioma. Cir Res 85: $992-999$

Mandriota SJ, Jussila L, Jeltsch M, Compagni A, Baetens D, Prevo R, Banerji S, Huarte J, Montesano R, Jackson DG, Orci L, Alitalo K, Christofori G, Pepper MS (2001) Vascular endothelial growth factor-C-mediated lymphangiogenesis promotes tumour metastasis. EMBO J 20: 672-682

Maula SM, Luukkaa M, Grenman R, Jackson D, Jalkanen S, Ristamaki R (2003) Intratumoral lymphatics are essential for the metastatic spread and prognosis in squamous cell carcinomas of the head and neck region. Cancer Res 63: 1920-1926

Nathanson SD, Zarbo RJ, Wachna DL, Spence CA, Andrzejewski TA, Abrams J (2000) Microvessels that predict axillary lymph node metastases in patients with breast cancer. Arch Surg 135: 584-593

Padera TP, Yun C, Kadambi A, Mouta Carreira C, Jain RK (2000) Local mechanism and VEGF-C alter peri-tumor lymphatic function. Proc Am Assoc Cancer Res 41: 88

Schoppmann SF, Bayer G, Aumayr K, Taucher S, Geleff S, Rudas M, Kubista E, Hausmaninger $H$, Samonigg $H$, Gnant $M$, Jakesz $R$, Horvat $R$, the Austrian Breast and Clorectal Cancer Study Group (2004) Prognostic value of lymphangiogenesis and lymphovascular invasion in invasive breast cancer. Ann Surg 240: $306-312$

Schoppmann SF, Birner P, Studer P, Breiteneder-Geleff S (2001) Lymphatic microvessel density and lymphovascular invasion assessed by anti-podoplanin immunostaining in human breast cancer. Anticancer Res 21: $2351-2356$

Skobe M, Hawighorst T, Jackson DG, Prevo R, Janes L, Velasco P, Riccardi L, Alitalo K, Claffey K, Detmar M (2001) Induction of tumour lymphangiogenesis by VEGF-C promotes breast cancer metastasis. Nature Med 7: $192-198$

Sleeman JP (2000) The lympn node as a bridgehead in the metastatic dissemination of tumors. Recent Results Cancer Res 157: 55-81

Stacker SA, Caesar C, Baldwin ME, Thornton G, Williams RA, Prevo R, Jackson DG, Nishikawa S, Kubo H, Achen MG (2001) VEGF-D promotes the metastatic spread of tumour cells via the lymphatics. Nature Med 7: $186-191$

Straume O, Jackson DG, Akslen LA (2003) Independent prognostic impact of lymphatic vessel density and presence of low grade lymphangiogenesis in cutaneous melanoma. Clin Cancer Res 9: 150-155

Weidner N, Semple JP, Welch WR, Folkman J (1991) Tumor angiogenesis and metastasis-correlation in invasive breast carcinoma. $N$ Engl J Med 324: $1-8$

Wigle JT, Oliver G (1999) Prox 1 function is required for the development of the murine lymphatic system. Cell 98: 769-778

Williams CSM, Leek RD, Robson AM, Banerji S, Prevo R, Harris AL, Jackson DG (2003) Absence of lymphangiogenesis and intratumoural lymph vessels in human metastatic breast cancer. J Pathol 200: 195-206 